\title{
ESTUDIO SOBRE EL NIVEL DE SALUD EN LOS ESTUDIANTES DE CIENCIAS DE LA UNIVERSIDAD DE EXTREMADURA (ESPAÑA)
}

\author{
Amalia Rodríguez Rodríguez \\ Fundación Sopeña \\ Badajoz. España \\ amaliar@telefonica.net \\ Eloísa Guerrero Barona \\ Universidad Extremadura \\ Badajoz. España \\ Juan Manuel Moreno Manso \\ Universidad Extremadura \\ Badajoz. España
}

Recepción Artículo: 22 enero 2020

Admisión Evaluación: 4 marzo 2020

Informe Evaluador 1: 1 abril2020

Informe Evaluador 2: 7 abril 2020

Aprobación Publicación: 20 abril 2020

\section{RESUMEN}

Dentro de la psicología positiva uno de los temas de investigación es la exploración en el bienestar de las personas y para ello se han realizado estudios sobre las fortalezas y las variables predictoras de la felicidad, entre ellas la salud percibida.

Los estudiantes universitarios se enfrentan a cambios en sus vidas y cambios en nuevos roles dirigidos hacia su futuro, así como a nuevas expectativas y horizontes.

El presente trabajo tiene como objetivo conocer la salud percibida en los estudiantes de ciencias de la Universidad de Extremadura. La salud percibida se considera un indicador de la salud general, se identifica con una parte del bienestar psicológico, posible indicador de una adaptación psicológica positiva a los grandes cambios. El estudio de dichas variables no va a permitir conocer la calidad de vida relacionada con el desempeño de sus funciones como estudiantes y de sus relaciones tanto con la universidad como con su entorno estudiantil.

Se ha analizado la variable salud y su relación con el género, edad y titulación. La muestra está compuesta por 503 alumnos de ciencias de la Universidad de Extremadura.

El instrumento empleado ha sido el Cuestionario de Salud General de Goldberg (GHQ12) Esta escala refleja preguntas relacionadas con la ausencia o presencia de síntomas de malestar psicológico como falta de sueño, alteraciones en el estado de ánimo, etc.

Los resultados indican que los varones presentan mayor percepción de salud que las mujeres, que no existe una relación indirecta entre edad del alumnado y su nivel de salud ni tampoco diferencias de salud del alumnado dependiendo de su titulación.

Palabras clave: salud; estudiantes universitarios; GHQ12 


\section{ESTUDIO SOBRE EL NIVEL DE SALUD EN LOS ESTUDIANTES DE CIENCIAS DE LA UNIVERSIDAD DE EXTREMADURA (ESPAÑA)}

\section{ABSTRACT}

Study on the level of health in science students at the university of Extremadura (Spain). Within positive psychology, one of the research topics is the exploration of people's well-being and for this, studies have been conducted on the strengths and predictive variables of happiness, including perceived health.

University students face changes in their lives and changes in new roles directed towards their future, as well as new expectations and horizons.

The present work has as objective to know the perceived health in the students of sciences of the University of Extremadura. Perceived health is considered an indicator of general health, it is identified with a part of psychological well-being, a possible indicator of a positive psychological adaptation to major changes. The study of these variables will not allow to know the quality of life related to the performance of their functions as students and their relationships with both the university and its student environment.

The health variable and its relationship with gender, age and degree have been analyzed. The sample is made up of 503 science students from the University of Extremadura.

The instrument used has been the Goldberg General Health Questionnaire (GHQ12) This scale reflects questions related to the absence or presence of symptoms of psychological distress such as lack of sleep, mood alterations, etc.

The results indicate that men have a higher perception of health than women, that there is no indirect relationship between the age of the students and their level of health, nor are there differences in the health of the students depending on their degree.

Keywords: health; university students; GHQ12

\section{ANTECEDENTES}

El equilibrio entre el organismo y el medio es, quizás, el modo más clásico de conceptualizar la salud. Galeno (129 d. C.) afirmaba que la salud es una situación de perfecto equilibrio y de armonía perfecta integrada por los principios de la naturaleza, de los humores que en nosotros existen, o simplemente la actuación sin ningún obstáculo de las fuerzas naturales. Esta definición clásica permanece hoy en día con ligeros cambios y transformaciones.

Se parte de un concepto de salud como ausencia de enfermedad. En esta línea surge la primera definición de la OMS y que posteriormente, en 1948, define la salud como un estado de completo bienestar físico, mental y social y no solamente la ausencia de enfermedad o dolencia.

Es una definición normativa y deontológica, útil para establecer líneas específicas de acción en políticas de salud.

En la década de los años 50 la Organización Panamericana de la Salud incluye también el concepto de medio ambiente en el que se mueve la persona. Posteriormente la OMS define la salud a través de unos componente 0 dimensiones como el estado de adaptación al medio (bilógico y /o sociocultural), el estado fisiológico de equilibrio, el equilibrio entre la forma y la función del organismo y la perspectiva biológica y social.

López, García y Dresch (2006) analizan las diferencias en salud autopercibida en hombres y mujeres teniendo en cuenta su situación laboral. Cuando se analiza en general las mujeres tienen peor salud que la población masculina, pero esto cambia cuando varían las condiciones de trabajo, incluso llegando a desaparecer. Las variables psicológicas predicen más la salud en las mujeres que en los varones, lo cual indica que la salud de las mujeres está íntimamente unida a la salud psicológica. Si continuamos con la relación entre la percepción de salud, bienestar psicológico y apoyo social percibido, Vivaldi y Barra (2012) realizan un estudio en población adulta en el cual comprobaron el bienestar psicológico presentaba mayores con el apoyo social percibido que con la percepción de salud, a su vez los hombre informaron de mayor bienestar psicológico y mejor percepción de salud que las mujeres.

Lao y Hueso (2014) aplican la ENSE (Encuesta Nacional de Salud de España, del Instituto Nacional de Estadística) y estudian la salud percibida, comprobando que a medida que aumenta la edad disminuye la percep- 
ción de salud y la tendencia general es que en ningún rango de edad existen cambios significativos en los veinte años de duración, no existiendo tampoco diferencias determinantes entre género, manteniendouna tendencia a la estabilidad. En edades superiores a nuestra muestra poblacional hallan otros datos pero no son relevantes para nuestra investigación.

La población estudiantil está sometida a una actividad con periodos particularmente estresantes, se enfrentan cada vea a más exigencias, desafíos y retos que les demandan una gran cantidad de recurso físicos y psicológicos, Estas situaciones les lleva al agotamiento físico y psicológico, llegando a desarrollar problemas de salud. Síntomas como cansancio, insomnio, agotamiento físico y mental, falta de concentración, dolores de espalda y cabeza y pocos hábitos de vida saludable correlacionan negativamente con el rendimiento académico, por el contrario salud mental, bajo estrés, y hábitos de vida saludable correlacionan positivamente con un medio alto rendimiento académico ( Ariño \&Fernández, 2015).

\section{OBJETIVOS}

En este estudio se pretende investigar si existen diferencias en el nivel de salud del alumnado de ciencias en función del género, edad, titulación. Buscamos identificar si las mujeres tienen niveles más elevados de salud que los varones, si a medida que aumenta la edad disminuye el nivel salud y si existen diferencias en la salud del alumnado encuestado dependiendo de su titulación.

\section{MÉTODO}

\section{Participantes}

La muestra objeto de estudio está compuesta por 503 estudiantes de las facultades de ciencias de Extremadura, siendo el $35.79 \%$ de la facultad de biología, el $12.52 \%$ de matemáticas, $29.42 \%$ de física, el $11.33 \%$ de químicas y el $10.93 \%$ de ciencias ambientales. El $50.33 \%$ son mujeres y el $49.67 \%$ son hombres. El rango de edad comprendido entre 18 y 22 años comprende el $84.4 \%$ del total del alumnado y el estado civil es de un $91 \%$ de solteros y el $8.4 \%$ viven en pareja o unión libre.

\section{Instrumentos}

- Cuestionario ad hoc para recoger datos sociodemográficos (sexo, edad, estado civil, beca, etc.) así como año de antigüedad en la carrera y año que cursa.

- El Cuestionario de Salud General de Goldberg (GHQ12) es elaborado por Goldberg, en la década de los 70, en el Hospital Universitario de Manchester, Inglaterra, con el fin de evaluar el grado de salud general.

- Es un cuestionario diseñado para ser aplicado en el ámbito sanitario médico, y se centra en la incapacidad para seguir llevando a cabo las funciones saludables normales y el origen de nuevos fenómenos del tipo del malestar psíquico. No es un cuestionario destinado a detectar psicosis funcionales, tipo esquizofrenia, ya que como cuestionario de screening o cribado no establece diagnósticos clínicos.

- Se han elaborado versiones cortas, de 30, 28 y 12 ítems que han mostrado utilidad en más de 50 estudios de validación. El resultado han sido un test con unas adecuadas propiedades psicométricas, con una buena sensibilidad, especificidad y fácil de administrar.

- El método de valoración es una escala tipo Likert, con valores de 0 a 3, siendo el máximo de 36 y el mínimo 0.El total se obtiene sumando las puntuaciones de cada ítem (García, 1999). La mayor ventaja que aporta este cuestionario es que está muy extendido y es de uso muy habitual lo que nos permite comparar resultados de muchos otros estudios y lugares. En cuanto a la fiabilidad y consistencia interna, los valores del Coeficiente alfa oscilan entre.82 y.93 (los más bajos corresponden a las versiones con menos ítems).

\section{Procedimientos}

Una vez seleccionados los instrumentos de medida y aplicación de las técnicas de muestreo se les pasó el cuestionario a todos los participantes. 


\section{ESTUDIO SOBRE EL NIVEL DE SALUD EN LOS ESTUDIANTES DE CIENCIAS DE LA UNIVERSIDAD DE EXTREMADURA (ESPAÑA)}

Todos los estudiantes completaron las pruebas en una única sesión en horario de clase y en presencia de la investigadora. Los estudiantes participaron de forma voluntaria, anónima y confidencial. Se les explicó que dicha información solo se emplearía con fines de investigación.

Los participantes completaron un cuestionario, elaborado para dicha investigación, que contiene la Escala de Satisfacción Vital (SWLS, Ed Diener et al 1985), la Escala de Felicidad, de Lyubomirsky y Lepper(1999) y el Cuestionario de Salud General de Goldberg(Goldberg,1970) entre otros cuestionarios más.

Todos los datos recogidos se codificaron y grabaron en soporte informático y posteriormente fueron analizados con el paquete estadístico SPSS 20.0 versión para Windows.

\section{RESULTADOS}

En la tabla 1 se muestran la media de salud percibida en función del género. Podemos observar que los hombres tienen una ligera mayor percepción de salud que las mujeres.

Tabla 1:

Media del total de salud dependiendo del genero

\begin{tabular}{|c|c|c|c|c|c|c|c|c|}
\hline & & & Media & $\begin{array}{r}\text { Median } \\
\mathrm{a}\end{array}$ & Moda & $\begin{array}{l}\text { Desviaci } \\
\text { ón } \\
\text { típica }\end{array}$ & $\begin{array}{r}\text { Mínim } \\
\text { o }\end{array}$ & $\begin{array}{r}\text { Máxim } \\
\text { o }\end{array}$ \\
\hline \multirow{2}{*}{ Sexo } & Mujer & $\begin{array}{l}\text { TOTAL } \\
\text { SALUD }\end{array}$ & 3.54 & 3.50 & 3.50 & .85 & 1.25 & 5.00 \\
\hline & $\begin{array}{l}\text { Hombr } \\
\text { e }\end{array}$ & $\begin{array}{l}\text { TOTAL } \\
\text { SALUD }\end{array}$ & 3.78 & 3.75 & 4.00 & $\begin{array}{l}.8 \\
7\end{array}$ & 1.00 & 5.00 \\
\hline
\end{tabular}

A tenor del gráfico anterior, parece que los hombres muestran un Nivel deSalud más elevado que las mujeres.

Tabla 2:

Prueba U de Mann Withney

\begin{tabular}{lr}
\multicolumn{2}{c}{ Estadísticos de contraste $^{\mathbf{a}}$} \\
\hline & TOTA \\
& L \\
& SALU \\
& D \\
\hline U de Mann-Whitney & 21916.500 \\
W de Wilcoxon & 48944.500 \\
Z & -3.263 \\
Sig. asintót. (bilateral) & .001 \\
\hline a. Variable de agrupación: Sexo \\
\hline
\end{tabular}

En la Tabla 2 se observa una $p=.001$ por lo que se rechaza Ho, trabajando con un Nivel de Confianza del $95 \%$. Es decir, existen diferencias entre hombre y mujeres en lo que a la Salud percibida se refiere.

Ello, unido a los resultados descriptivos, nos indica que los hombres presentan un nivel significativamente más elevado de salud que las mujeres, por lo que se acepta la hipótesis de trabajo planteada.

El segundo planteamiento es que existe una relación indirecta entre la edad del alumnado y la salud. 
Tabla 3:

Prueba K-S (Principio de Normalidad) sobre total salud y edad del alumnado

\section{SALUD}

\begin{tabular}{llr}
\hline \hline & & TOTAL SALUD \\
\hline $\mathrm{N}$ & Media & 461 \\
& & 3.6556 \\
Parámetros normalesa,b & & \\
& Desviación típica & .87012 \\
& Absoluta & .096 \\
Diferencias más & Positiva & .061 \\
extremas & Negativa & \\
& & -.096 \\
Z de Kolmogorov- & & 2.068 \\
Smirnov & & .000 \\
Sig. asintót. (bilateral) & & \\
\hline
\end{tabular}

En la Tabla 3 se presenta el resultado obtenido de la prueba K-S para Salud, obteniendo $p<.05$, por lo que se rechaza el Principio de Normalidad en esta serie de datos. Se aplica por tanto una prueba estadística no paramétrica, concretamente la Prueba Rho de Spearman.

Tabla 4:

Correlación de Spearman entre total de salud y edad del alumnado

\section{Correlaciones}

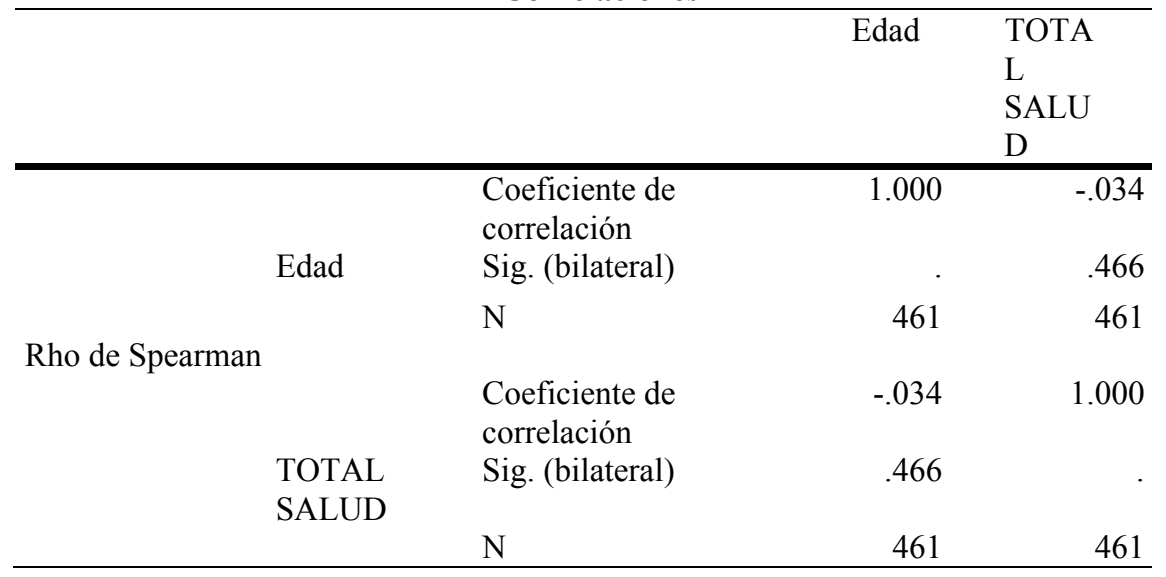

La tabla 4 nos muestra que la relación entre estas variables es muy débil (próxima a 0) y negativa. № así, al remitirnos al $p$ valor asociado y, puesto que éste es mayor que $\alpha(.05)$, se acepta $\mathrm{Ho}$, lo que indica que no existe relación entre las variables contrastadas, por tanto, se rechaza la hipótesis de trabajo.

Nuestra última hipótesis es conocer si existen diferencias de salud percibida del alumnado encuestado dependiendo de su titulación. 


\section{ESTUDIO SOBRE EL NIVEL DE SALUD EN LOS ESTUDIANTES DE CIENCIAS DE LA UNIVERSIDAD}

DE EXTREMADURA (ESPAÑA)

Tabla 5:

Estadísticos descriptivos Total Salud

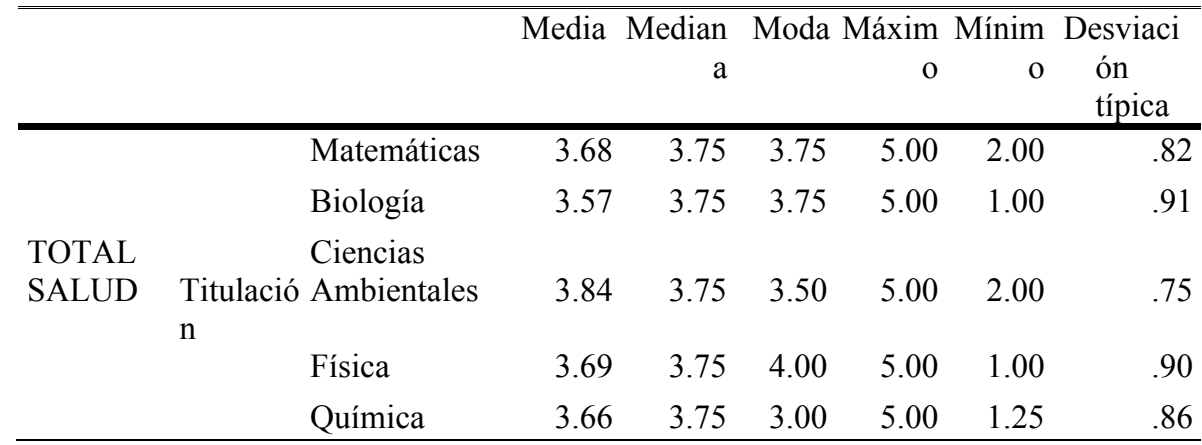

De esta forma se observa cómo las puntuaciones de todas las titulaciones son aparentemente similares, destacando que el alumnado de Ciencias Ambientales es el que parece presentar un nivel más elevado de Salud.

Los datos no parecen seguir la dirección marcada en la hipótesis de trabajo, no obstante no diremos nada concluyente hasta realizar el pertinente análisis inferencial.

Tras la realización de la prueba KS (Kolmogorov-Smirnov) para una muestra se obtiene una $p=.268$, mayor que $\alpha$ (.05). Por tanto, se acepta la hipótesis nula (en adelante Ho) trabajando con un Nivel de Confianza del 95\%. Por tanto, se acepta el Principio de Normalidad en esta seria de datos.

No proseguiremos realizando los contrastes restantes, al no aceptarse el principio anterior, por lo que utilizaremos directamente un modelo no paramétrico. Puesto que trabajamos con más de 2 grupos (5 titulaciones), aplicaremos la Prueba $\mathrm{H}$ de Kruskal Wallis.

Tabla 6:

Prueba Kruskal Wallis total salud

\begin{tabular}{lr}
\multicolumn{2}{c}{ Estadísticos de contraste $^{\mathbf{a}, \mathbf{b}}$} \\
\hline & TOTA \\
& L \\
& SALU \\
& D \\
\hline Chi-cuadrado & 3.385 \\
gl & 4 \\
Sig. asintót. & .496 \\
\hline \hline a. Prueba de Kruskal-Wallis \\
b. Variable de agrupación: \\
Titulación
\end{tabular}

Tras aplicar esta prueba (Tabla 6), se obtiene una $p=.496$, mayor que (.05). Se acepta Ho, lo que indica que no existen diferencias significativas entre las medias de las series de datos, trabajando con un Nivel de Confianza del $95 \%$.

Por tanto, puesto que las puntuaciones son estadísticamente similares, rechazamos la hipótesis de trabajo. 


\section{CONCLUSIONES}

En este trabajo de investigación nos propusimos conocer los niveles de salud percibida, que tienen nuestros alumnos de las facultades de ciencias de la Universidad de Extremadura.

Numerosos estudios han puesto de manifiesto que la salud está relacionada con otras variables como el apoyo social percibido, nivel de autonomía personal o rendimiento académico. Es diferente la percepción de salud dependiendo de la edad. En nuestro estudio hemos encontrado que no existe una relación indirecta entre las variables edad y salud. Estos resultados no coinciden con los obtenidos por Lao \& Hueso (2014) que al aplicar la Encuesta Nacional de Salud de España (ENSE) obtienen que cuando aumenta la edad disminuye la percepción de salud posiblemente esto es debido a que la muestra de nuestra investigación tiene un rango de edad corto y este autor utiliza un rango de edad más amplio.

García, Dresch y López (2006) analizan las diferencias en salud autopercibida en hombres y mujeres teniendo en cuenta su situación laboral. Cuando se analiza en general las mujeres tienen peor salud que la población masculina, esto iría en la misma línea de nuestra investigación.

Para finalizar según nuestros resultados no encontramos relación indirecta entre edad y percepción de salud, así como tampoco existen diferencias dependiendo de la titulación. Respecto al género los varones tienen mejor percepción de salud que las mujeres.

\section{REFERENCIAS BIBLIOGRÁFICAS}

Ariño, A. P., \& Fernández, J. G. (2015). Rendimiento académico y correspondencias con indicadores de salud física y psicológica. Sportis. Scientific Journal of School Sport, Physical Education and Psychomotricity, 1(2), 164-181.

García Viniegra, C. R. (1999). Manual para la utilización del cuestionario de salud general de Goldberg: Adaptación cubana. Revista Cubana de Medicina General Integral, 15(1), 88-97

Lao, J. G., \& Hueso, A. D. C. (2014). ¿Informa la salud autopercibida sobre las condiciones objetivas de salud? Algunas conclusiones a partir del análisis demográfico de microdatos de la Encuesta Nacional de Salud. Estadística Española, 56(183), 61-76.

López, M. P. S., García, M. E. A., \& Dresch, V. (2006). Ansiedad, autoestima y satisfacción autopercibida como predictores de la salud: diferencias entre hombres y mujeres. Psicothema, 18(3), 584-590.

Organización Mundial de la Salud. http://www.euro.who.int/en/home

Organización Panamericana de la Salud. https://www.paho.org/hq/index.php?lang=es

Vivaldi, F., \& Barra, E. (2012). Bienestar psicológico, apoyo social percibido y percepción de salud en adultos mayores. Terapia psicológica, 30(2), 23-29. 
